Revue d'histoire de l'Amérique française

Q4. REVUE D'HISTOIRE DE L'AMÉRIQUE FRANÇAISE

\title{
Le livre et la lecture dans la noblesse canadienne 1670-1764
}

\section{Mario Robert}

Volume 56, numéro 1, été 2002

URI : https://id.erudit.org/iderudit/007215ar

DOI : https://doi.org/10.7202/007215ar

Aller au sommaire du numéro

\section{Éditeur(s)}

Institut d'histoire de l'Amérique française

\section{ISSN}

0035-2357 (imprimé)

1492-1383 (numérique)

Découvrir la revue

\section{Citer cet article}

Robert, M. (2002). Le livre et la lecture dans la noblesse canadienne 1670-1764. Revue d'histoire de l'Amérique française, 56(1), 3-27.

https://doi.org/10.7202/007215ar

\section{Résumé de l'article}

Depuis quelques années, on assiste à un regain d'intérêt pour la noblesse canadienne en Nouvelle-France. Les attributs et comportements démographiques de ce groupe social sont maintenant connus, alors que leurs pratiques culturelles nous sont peu familières. Afin de mieux cerner certaines de ces pratiques, cet article étudie la présence du livre dans la noblesse canadienne entre 1670 et 1764 . À partir d'un corpus documentaire de 276 actes notariés, principalement des inventaires après décès, et selon les méthodes quantitatives développées par les historiens français du livre, le texte identifie le nombre et l'importance des collections d'imprimés, les lieux de conservation et la nature de ces collections. Il propose aussi d'examiner sommairement l'usage que les nobles font de leurs livres. Cet article vise finalement à démontrer que les nobles canadiens se distinguent de l'ensemble de la population de la Nouvelle-France en matière de culture livresque. 


\section{Le livre et la lecture dans la noblesse canadienne $1670-1764^{1}$}

MARIO ROBERT

Section des archives

Ville de Montréal

RÉSUMÉ - Depuis quelques années, on assiste à un regain d'intérêt pour la noblesse canadienne en Nouvelle-France. Les attributs et comportements démographiques de ce groupe social sont maintenant connus, alors que leurs pratiques culturelles nous sont peu familières. Afin de mieux cerner certaines de ces pratiques, cet article étudie la présence du livre dans la noblesse canadienne entre 1670 et 1764 . À partir d'un corpus documentaire de 276 actes notariés, principalement des inventaires après décès, et selon les méthodes quantitatives développées par les historiens français du livre, le texte identifie le nombre et l'importance des collections d'imprimés, les lieux de conservation et la nature de ces collections. II propose aussi d'examiner sommairement l'usage que les nobles font de leurs livres. Cet article vise finalement à démontrer que les nobles canadiens se distinguent de l'ensemble de la population de la Nouvelle-France en matière de culture livresque.

ABSTRACT - Recent years have seen increased interest in the Canadian nobility in New France. The attributes and demographic habits of this social group are now familiar to us while their cultural practices are still little known. In order to better understand some of these elements, this article deals with book ownership among the Canadian nobility between 1670 and 1764. Using a documentary corpus of 276 notarized acts, principally post-mortem inventories, and following the quantitative methods developed by French scholars specializing in the history of reading, this article identifies the number and size of

1. Je remercie ma directrice de mémoire, Sylvie Dépatie, pour ses commentaires, de même que les évaluateurs de la revue. 
collections of printed materials, the places in which they were kept and the nature of these collections. It briefly considers how Canadian noblemen use their books and shows that they differ from the general population of New France as far as books are concerned.

La seule possession d'un livre implique une situation sociale et une certaine richesse intellectuelle Alberto Manguel ${ }^{2}$

I a Nouvelle-France, à l'instar de sa métropole, disposait d'un L second ordre dont on connaît maintenant les attributs et les comportements démographiques ${ }^{3}$. Toutefois, les habitudes culturelles de ce groupe social sont méconnues. L'historiographie, s'appuyant surtout sur la correspondance officielle des dirigeants de la Nouvelle-France, a toujours conclu que le niveau de culture de cette noblesse, souvent qualifiée d'indigente, était peu élevé. Ainsi, Francis Parkman, le premier, dit d'eux qu'ils sont pauvres sans être miséreux, peu cultivés mais aspirant à un mode de vie calqué sur l’aristocratie métropolitaine:

The beggared noble of the early time became a sturdy country gentleman; poor, but not wretched; ignorant of books, except possibly a few scraps of old rusty Latin picked up in a Jesuit school; hardy as the hardiest woodsman, yet never forgetting his quality of gentilhomme; scrupulously wearing its badge, the sword, and copying as well as he could the fashions of the court, which glowed on his vision across the sea in all the effulgence of Versailles, and beamed with reflected ray from the chateau of Quebec ${ }^{4}$.

Louise Dechêne qualifie cette noblesse de "gentilhommerie arrogante, ignorante et incapable de pourvoir à ses besoins ${ }^{5}$ ». À partir d'un

2. Alberto Manguel, Une histoire de la lecture (S.1., Leméac, coll. "Actes Sud», 1998), 253.

3. Depuis la parution de La Noblesse de Nouvelle-France: familles et alliances de Lorraine Gadoury (Montréal, Hurtubise HMH, 1992). Être noble en Nouvelle-France signifie qu'il «faut être qualifié d'écuyer dans les actes - être donc perçu et accepté en tant que noble par son milieu —, vivre noblement — c'est-à-dire ne pas déroger en exerçant un travail manuel —, et servir le Roi; dans une majorité des cas, dans la colonie, les nobles servent en combattant, mais d'autres formes de service sont considérées: administration, magistrature... », L. Gadoury, La noblesse de Nouvelle-France..., 20.

4. Francis Parkman, France and England in North America, I: Pionners of France in the New World; The Jesuits in North America in the Seventeenth Century; La Salle and the Discovery of the Great West; The Old Regime in Canada) (S.1., The Library of Americas, 1983), 1287. Cette citation est tirée de l'ouvrage The Old Regime in Canada publié une première fois en 1874.

5. Louise Dechêne, Habitants et marchands de Montréal (Montréal, Boréal, 1988), 383. 
nombre restreint d'inventaires après décès (17), elle montre que la plupart sont endettés à cause d'un mode de vie somptueux et que quelques familles possèdent une quantité limitée de livres ainsi qu'un ou deux tableaux religieux. De son côté, Marcel Trudel prétend que leur niveau culturel n'est pas différent des autres groupes sociaux de la NouvelleFrance, alors que Christopher Moore souligne que «[r]ares, semble-t-il, sont les membres de cette élite coloniale [les nobles] qui s'intéressent à la vie intellectuelle ou aux lettres ${ }^{6}$ ». Nous nous proposons donc de faire le point sur ce postulat en nous consacrant aux habitudes culturelles de la noblesse canadienne. Nous tenterons ainsi de cerner partiellement leur niveau de culture à partir d'une étude de la présence du livre et de la lecture au sein de la noblesse canadienne entre 1670 et 1764 comme l'ont fait nombre d'historiens français à partir des années 1960 et d'historiens québécois à partir de la décennie suivante à l'intérieur d'une branche spécifique de l'histoire culturelle : l'histoire quantitative du livre. Notre enquête nous permettra d'identifier le nombre et l'importance des collections d'imprimés, les lieux de conservation et la nature de ces collections. À titre d'essai, nous présenterons sommairement les pratiques de lecture des nobles canadiens, ce que l'historien français HenriJean Martin identifie comme étant les «signes de lecture». Finalement, nous nous demanderons si, dans le domaine de la culture livresque, les nobles se distinguent de l'ensemble de la population canadienne en Nouvelle-France.

\section{DÉMARCHE ET MÉTHODOLOGIE}

Cette recherche s'inscrit dans la foulée des études quantitatives sur la présence du livre et des bibliothèques privées réalisées en France après 1955 et au Québec à compter des années 1970. Des historiens français tels que Henri-Jean Martin, Lucien Febvre, François Furet ou Alphonse Dupront s'intéressent alors à la production, à la diffusion et à la consommation du livre par l'étude des éditeurs, des libraires et bien sûr des lecteurs. Au Québec, le Groupe de recherche en histoire du livre, créé en 1974 et dirigé par l'historien Claude Galarneau, se donne pour mission d'étudier la production, la diffusion et la consommation de l'imprimé dans la ville de Québec pour la période 1764 à $1860^{7}$. Cette démarche est

6. Christopher Moore, «La Nouvelle-France et ses rivales, 1600-1760», dans Craig Brown, dir., Histoire générale du Canada (Montréal, Boréal, 1988), 189.

7. Claude Galarneau, «Livre et société à Québec (1760-1859) : état des recherche», dans Yvan Lamonde, dir., L'imprimé au Québec: aspects historiques (18-20 siècles) (Québec, Institut québécois 
remise en question dans les années 1980 par les historiens français qui l'ont créée. Avec Roger Chartier et Henri-Jean Martin en tête, ils affirment que les «historiens du livre ont trop longtemps sacrifié à la statistique $^{8}$ " et que l'inventaire après décès n'indique que la "pesée du livre». Pour eux, il faut mettre de l'avant une diversité de sources pour identifier les «signes de lectures" car, et c'est là leur leitmotiv, un livre possédé n'est pas nécessairement un livre lu. Chartier et Martin sont néanmoins conscients que ce renouvellement de l'histoire du livre a été rendu possible grâce au nombre important d'études quantitatives qui ont permis l'écriture de synthèses sur la production, sur la diffusion et sur la consommation du livre?.

En ce qui concerne la Nouvelle-France, toute étude sur la culture livresque doit commencer par l'analyse quantitative de la présence du livre dont la source essentielle est l'inventaire après décès, et ce, malgré ses lacunes. En effet, l'inventaire ne couvre pas toutes les communautés de biens; il est dressé, en dépit de la prescription légale, plusieurs années après le décès de l'une des parties; tandis que la qualité de la description est variable d'un notaire à l'autre. Mais en raison de l'absence de catalogues imprimés et vu le côté aléatoire de la recherche des «signes de lectures», qui demande une consultation large de différentes sources telles que la correspondance, les mémoires ou même les documents judiciaires ${ }^{10}$, l'inventaire après décès constitue la source quantitative la plus commode pour connaître l'accès aux livres en Nouvelle-France.

La démarche que nous avons adoptée consiste donc à repérer les inventaires après décès (et parfois des procès-verbaux de vente de biens)

de recherche sur la culture, 1983). Deux mémoires couvriront la période 1760 à 1819: Égide Langlois, Livres et lecteurs à Québec, 1760-1799, mémoire de maîtrise, Université Laval, 1984, 112 p. ; Yvan Morin, Les niveaux de culture à Québec 1800-1819. Étude des bibliothèques privées par les inventaires après décès, mémoire de maitrise, Université Laval, 1979, 140 p. S'inspirant des travaux effectués à l'Université Laval, une étude de la consommation du livre dans la ville de Québec durant le Régime français sera réalisée par Gilles Proulx pour le compte de Parcs Canada (Gilles Proulx, Loisirs québécois: des livres et des cabarets 1690-1760 (Québec, Service canadien des parcs, 1987).

8. Henri-Jean Martin et Bruno Delmas, Histoire et pouvoirs de l'écrit (Paris, Albin Michel, 1996), 309.

9. En 1992, Roger Chartier déclarait que «[1]a démarche, collectivement assumée (y compris par l'auteur de ce texte), a accumulé un savoir sans lequel d'autres interrogations auraient été impensables. » Cité dans Roger Chartier, L'ordre des livres: lecteurs, auteurs, bibliothèques en Europe entre XIV et XVIII siècle (Aix-en-Provence, Alinéa, 1992), 18.

10. On retrouve ainsi des exemples de «signes de lectures» dans des témoignages judiciaires recensés par Gilles Proulx, Loisirs québécois : des livres et des cabarets 1690-1760, 109. 
de chaque membre de la noblesse canadienne ${ }^{11}$ avant $1764^{12}$, dans la banque de données Parchemin, à partir de la liste des familles établie par Lorraine Gadoury dans son ouvrage La Noblesse de Nouvelle-France: familles et alliances. Différentes stratégies de recherche ont ainsi permis d'identifier 243 inventaires après décès et 31 procès-verbaux de vente. Toutefois, il existe des actes notariés non recensés dans Parchemin. Ces documents se retrouvent dans la Collection de pièces judiciaires et notariées (NF-25) disponible aux Archives nationales du Québec à Québec ${ }^{13}$. La consultation de cet outil a permis d'ajouter deux inventaires ${ }^{14}$. Le corpus documentaire final s'élève donc à 276 actes notariés dépouillés dans 82 greffes de notaires des régions de Montréal, de Québec et de TroisRivières. De ce nombre, 251 sont retenus pour l'étude; les 25 autres sont en effet des procès-verbaux de vente dont nous possédons déjà l'inventaire. Par conséquent, nous possédons l'ensemble des inventaires après décès disponibles pour les membres de la noblesse canadienne entre 1670 et 1764 .

Toute analyse quantitative de la présence du livre doit, selon Daniel Roche, entreprendre "trois tests»: "celui de la présence ou de l'absence de livres, celui de leur nombre et de l'importance des collections, celui de la localisation dans l'habitation ${ }^{15}$ ». Nous commencerons donc par l'examen de ces trois éléments ainsi que de la place qu'occupe le livre chez les nobles selon leur fortune et leur occupation. Nous analyserons

11. La noblesse n'étant transmissible que par les hommes, seules, parmi les femmes, ont été retenues celles mariées à des nobles ainsi que les célibataires.

12. Toute personne voulant retourner en France avait jusqu'au 10 août 1764, date à laquelle prenait fin le régime militaire, pour quitter la colonie.

13. Pierre-Georges Roy, Inventaire d'une collection de pièces judiciaires, notariales, etc. conservées aux archives judiciaires de Québec (Beauceville, La Compagnie L’Éclaireur, 1917), 585 p., 2 vol.

14. Nous sommes conscients que des inventaires après décès de nobles peuvent exister dans d'autres fonds ou séries d'archives. Soulignons, par exemple, le très riche inventaire des livres de Thomas-Jacques Taschereau conservé dans le fonds Famille Taschereau (ANQ, Centre de Québec) alors que son inventaire après décès se trouve dans les archives coloniales françaises (disponible ici sur microfilm). Nous connaissons aussi, par divers ouvrages, les grandes bibliothèques constituées par deux fils de Pierre Boucher: les curés Philippe et Nicholas Boucher. Toutefois, nous nous en sommes tenu aux inventaires que nous avons extraits des deux principales sources d'information pour que les résultats de notre analyse ne soient pas affectés par l'inclusion d'inventaires connus précisément en raison de l'ampleur exceptionnelle des bibliothèques qu'ils décrivent, ampleur qui risquerait de donner des statistiques disproportionnées par rapport à la bibliothèque de taille courante chez le noble canadien.

15. Daniel Roche, Les Républicains des lettres: gens de culture et Lumières au XVIII siècle (Paris, Fayard, 1988), 91. 
ensuite les grands thèmes ou catégories littéraires des livres possédés par les nobles à partir du plan de classification élaboré par François Furet ${ }^{16}$.

\section{PRÉSENCE DU LIVRE DANS LES INVENTAIRES APRÈS DÉCÈs}

Les nobles canadiens sont familiers avec l'écrit. Leur position élitaire dans la société, leurs activités civiles, militaires ou même commerciales les obligent à lire et à écrire. Leurs inventaires après décès renferment d'ailleurs un bon nombre de titres et de papiers. De plus, rares sont les survivants du couple qui ne savent pas signer. Sur 115 actes, où l'on rencontre un conjoint survivant, seules quatre épouses ne sont pas en mesure d'apposer leur nom ${ }^{17}$. Lorraine Gadoury n'affirme-t-elle pas que les nobles et les marchands «se distinguent du reste de la population canadienne du xviII siècle: ils savent lire et écrire ${ }^{18}$ "? Dès lors, on devrait s'attendre à une plus grande concentration de livres ou d'imprimés chez ces deux groupes sociaux. Dans la période comprise entre 1670 et $1764,38 \%$ des inventaires après décès des nobles canadiens contiennent des livres ${ }^{19}$, soit 96 des 251 actes sélectionnés. Pour les trois dernières décennies du $\mathrm{xvII}^{\mathrm{e}}$ siècle, le taux de présence du livre est de $33 \%$, soit 10 des 30 actes disponibles, alors qu'il s'élève à $39 \%$ pour le XVIII ${ }^{\mathrm{e}}$ siècle (86 actes sur 221). Mais lorsqu'on sélectionne les années entre 1701 et 1750 , le taux atteint $44 \%$, tandis qu'il chute à $28 \%$ à la fin du régime français (voir tableau 1). La baisse observée à partir de 1751 est-elle liée à la guerre de la Conquête et à ses conséquences? La chute

16. Cette grille est souvent identifiée comme étant la nomenclature de la Bibliothèque royale du XviII siècle. François Furet, "La "librairie" du royaume de France au $18^{\mathrm{e}}$ siècle", dans Geneviève Bollème et al., Livre et société dans la France du XVIII siècle (Paris, Mouton, 1965), 3-32.

17. La valeur de la signature ne fait pas l'unanimité comme critère d'alphabétisation; mais, comme le souligne Michel Verrette, "[j]usqu’à présent, en histoire de l’alphabétisation, la meilleure trace identifiée et qui a été l'objet de la critique la plus sérieuse demeure la signature». Michel Verrette, L'alphabétisation au Québec 1660-1900: en marche vers la modernité culturelle (Québec, Septentrion, 2002), 49. Toutefois, et la littérature le confirme, l'assimilation de la lecture et de l'écriture n'est pas nécessairement certifiée par la signature d'un acte car «[e]n raison de l'ordre des apprentissages scolaires - on ne vient théoriquement à l'écriture qu'une fois la lecture acquise, on peut savoir lire sans pour autant savoir écrire ou signer. On peut tout aussi bien signer sans savoir écrire». François Melançon, "Façonner et surveiller l'intime: lire en Nouvelle-France», dans Manon Brunet et Serge Gagnon, dir., Discours et pratiques de le l'intime (Québec, Institut québécoise de recherche sur la culture, 1993), 20.

18. Lorraine Gadoury, La famille dans son intimité: échanges épistolaires au sein de l'élite canadienne du XVIII siècle (Montréal, Hurtubise HMH, 1998), 21.

19. Lorsqu'un inventaire mentionne que les livres ne sont pas décrits parce qu'ils sont réservés à l’homme en vertu du contrat de mariage, nous rangeons la personne concernée parmi les possesseurs de livres. 
TABLEAU 1

\section{Présence du livre dans les inventaires après décès des nobles canadiens 1670- 1764}

\begin{tabular}{lccc}
\hline Années & Actes & Actes avec livres & $\%$ \\
\hline $1670-1700$ & 30 & 10 & 33 \\
$1701-1764$ & 221 & 86 & 39 \\
$170 \mid-1750$ & 153 & 67 & 44 \\
$175 \mid-1764$ & $25 \mid$ & 96 & 28 \\
$1670-1764$ & 68 & 19 & 38 \\
\hline
\end{tabular}

des proportions qui se poursuit pour les années 1761-1764 est-elle attribuable au départ des nobles les plus lettrés ou les plus riches? On ne peut présentement qu'avancer l'hypothèse. Cette baisse du nombre d'inventaires avec livres n'est pas propre à la noblesse canadienne puisqu'elle touche l'ensemble de la population de la ville de Québec après 1760. Entre l'étude de Gilles Proulx pour la période 1690-1760 et celle d'Égide Langlois pour la période 1760-1799, la chute est de $5 \%$.

Le pourcentage global obtenu par la noblesse canadienne est supérieur à celui que l'on rencontre pour la population de la ville de Québec entre 1690 et 1760 . À Québec, $31 \%$ des inventaires après décès contiennent des livres comparativement à $38 \%$ pour les nobles canadiens. Il est difficile de comparer nos résultats avec ceux obtenus par les noblesses de France en raison de la disparité des périodes visées par les études françaises: 1697-1698 pour les villes de l'Ouest de la France, $1750-1759$ pour Paris et 1750-1780 pour Lyon. Tout au plus pouvons-nous constater qu'un peu moins de la moitié des nobles bretons au xviII siècle possèdent des livres, ce qui est supérieur à ce qu'obtient la noblesse canadienne, tandis qu'à Paris et à Lyon, ce sont $44 \%$ des inventaires qui mentionnent des livres ${ }^{20}$.

Les études françaises et québécoises du livre ont généralement fait état d'une corrélation entre la richesse et la présence du livre de même qu'entre la fortune et le nombre d'ouvrages. Pour Roger Chartier et Daniel Roche, "la proportion des propriétaires de livres croît avec l'élévation des niveaux de fortune [...] L'état ou la fortune déterminent aussi 
le nombre de livres possédés ${ }^{21}$. "En répartissant les fortunes nobiliaires ${ }^{22}$ canadiennes en six échelons ${ }^{23}$, on remarque que $53 \%$ d'entre elles s'élèvent à 1001 livres tournois et plus ${ }^{24}$. Si l'on soustrait, en raison de l'inflation, les 21 inventaires rédigés entre 1758 et 1762, on arrive à des conclusions similaires. Il faut cependant relativiser l'ensemble des chiffres obtenus et s'interroger sur la valeur totale de biens décrits dans l'inventaire. Comme le souligne Michel Marion, ces chiffres «ne peuvent être qu'indicatifs $[. .$.$] parce que trop d'éléments manquent parfois dans la$ source pour une évaluation ${ }^{25}$ ». L'absence de prisée pour les biens immobiliers en est un. Lorraine Gadoury affirme, pour la famille d'Ailleboust, que les ventes de terrains ou d'emplacements permettent de pallier le faible niveau de fortune ou même l'endettement élevé constaté dans l'inventaire après décès car "la terre semble [...] être pour les nobles canadiens un fondement essentiel de la fortune ${ }^{26} »$. Pour revenir à la présence du livre, on observe que $43 \%$ des inventaires de plus de 1001 livres en mentionnent, contre $31 \%$ pour les inventaires valant 1000 livres et moins. Toutefois, le pourcentage obtenu par les fortunes les plus élevées (10 001 livres et plus) est inférieur de $4 \%$ à celui des fortunes les plus modestes (1-500 livres), ce qui déroge donc des conclusions habituelles des historiens du livre.

On peut cependant établir des liens entre l'occupation et la présence de livres. Les inventaires de $39 \%$ des nobles ayant des fonctions militaires contiennent des imprimés contre $25 \%$ pour les nobles ne portant que le titre d'écuyer. Le taux le plus élevé, il ne faut pas s'en surprendre, concerne les conseillers royaux, puisque la consultation d'ouvrages juridiques, sur une base quotidienne, est essentielle à leur tâche. C'est pourquoi l'on retrouve des livres chez eux bien qu'il existe «une collection

21. R. Chartier et D. Roche, ibid., 404.

22. Les biens mobiliers, le numéraire et les créances ont été inclus dans le calcul des fortunes; ont été soustraites les dettes.

23. Ces échelons sont: 0 livres, 1-500 livres, 501-1000 livres, 1001-5000 livres, 5001-10 000 livres, 10001 livres et plus. La livre canadienne, qui vaut $25 \%$ de moins que la livre française, a cours au Canada des débuts de la colonies jusqu'en 1717. Pour des raisons d'uniformité, les valeurs des inventaires ont été transformées pour cette période en livres françaises. Entre 1717 et 1720 , nous avons converti les valeurs des inventaires qui étaient indiquées en «monnaye du pays ".

24. Lorsque l'on ne calcule que les actifs, la proportion grimpe à $71 \%$.

25. Michel Marion, Les bibliothèques privées à Paris au milieu du XVIII siècle (Paris, Bibliothèque nationale, 1978), 75.

26. L. Gadoury, La famille dans son intimité, op. cit., 36. 
d'ouvrages de droit, logée dans les bureaux du Conseil supérieur ${ }^{27}$ ». Sur 17 nobles portant ce titre dans l'acte notarié, 13 possèdent des livres (76\%). Finalement, pour les 43 chevaliers de l'Ordre royal et militaire de Saint-Louis, créé en 1693, les livres sont présents dans $46 \%$ de leurs inventaires, un chiffre supérieur à la moyenne et équivalent aux pourcentages affichés par les noblesses parisienne et lyonnaise.

Notons cependant que l'absence de livres dans les inventaires ne signifie pas pour autant que les nobles n'en possèdent pas. Il faut en effet envisager la possibilité que les livres aient été transmis du vivant du possesseur ou peu avant son décès. Par exemple, on ne rencontre aucun livre dans l'inventaire de l'ingénieur Gaspard Chaussegros de Léry décédé en 1756. Pourtant, un ingénieur se doit de posséder certains livres dans l'exercice de ses fonctions. En outre, l'importance qu'accorde ce noble aux livres est connue. En 1751, il achète une géographie de Nolin. Tout au long de sa carrière, il souhaite faire publier un traité sur les fortifications (huit tomes et 132 planches) qu'il a écrit avant son passage au Canada ${ }^{28}$. Les autorités françaises refusent en raison du coût élevé de la gravure et de l'impression des planches ${ }^{29}$. Chaussegros de Léry a-t-il cédé, de son vivant, ses livres à son fils, lui aussi ingénieur et qu'il avait par ailleurs formé ${ }^{30}$ ? On peut le penser puisqu'à la mort de ce dernier en 1797, on retrouve des ouvrages de Bernard Forest de Bélidor $^{31}$, de même que des livres de mécanique et de mathématiques ${ }^{32}$.

27. Antonio Drolet, Les bibliothèques canadiennes 1604-1960 (Montréal, Le Cercle du livre de France, 1965), 25.

28. Nous connaissons l'existence de lettres sur ce sujet en 1727, 1728, 1739 et 1754 (voir Pierre-Georges Roy, Inventaire des papiers de Léry (Québec, Archives de la province de Québec, 1939-1940), I: 184 et 192; II : 6-7 et 129.

29. Ce texte intitulé Traité de fortification divisé en huit livres où sont montrées les méthodes de quelques ingénieurs qui ont écrit de la fortification et plusieurs nouvelles manières de fortifier et disposer les ouvrages de dehors, retranchements des armées, attaques et défenses des places (s.1., s.é., 1714), 444 p., est resté à l'état de manuscrit et il est aujourd'hui conservé aux Archives nationales du Canada dans le Fonds Gaspard Chaussegros de Léry.

30. "Le fils aisné de cet officier est un jeune homme de 18 ans auquel le Pere a donné tous ses soins pour luy apprendre les fortiffication, l'arithmetique et le dessin. » Lettre de Beauharnois et Hocquart au président du Conseil de la Marine, 2 octobre 1738, dans Inventaire des papiers de Léry, I: 282-283.

31. On retrouve ainsi les ouvrages Architecture Hydraulique, ou l'Art de conduire, d'Elever, et de Menager les Eaux pour les Differens Besoins de la Vie dont la première édition paraît en 1737 de même La Science des Ingenieurs dans la Conduite des Travaux de Fortification et d'Architecture Civile paru en 1739.

32. «Bibliothèque de Joseph-Gaspard Chaussegros de Léry, ingénieur, capitaine dans les troupes de la Marine, chevalier de Saint-Louis ", Bulletin des recherches historiques, XL,9 (septembre 1934): 574. 
Il n'est pas impossible non plus que les livres soient exclus de l'inventaire parce qu'ils sont considérés comme biens propres, ne faisant pas partie de la communauté. Ceux de René-Louis Chartier (1701) et de son fils Eustache Chartier (1723) ne sont pas décrits dans l'inventaire car ils leur sont réservés en vertu de leur contrat de mariage. Dans ces deux cas, le notaire a bien pris soin de le mentionner dans l'inventaire. En 1723, lors de l'inventaire de sa communauté avec Marie-Françoise Renaud Davaine de Desméloizes, Eustache Chartier, seigneur de Lotbinière et conseiller du roi, s'oppose à la description de certains biens "attendu que par son contrat de mariage outre le preciput il doit remporter Ses habits, hardes et linges a Son usage et Sa bibliothèque ${ }^{33}$ ». Devenu veuf, il se fait prêtre et devient doyen du chapitre de la cathédrale de Québec. Il meurt en février 1749 et un nouvel inventaire comportant des livres est élaboré en mai. Ceux-ci se trouvent en deux endroits: à l'hôpital général où il vivait retiré depuis 1747, seul subsiste son bréviaire romain tandis que " 26 livres de différents autheurs et Sur différents Sujets, dont quelqu'un en latin et les autres en françois ${ }^{34}$ » sont rangés dans une armoire de bois de pin à cinq tiroirs chez son fils Michel, le futur marquis de Lotbinière.

La possibilité que les livres soient considérés comme biens propres semble se vérifier dans le cas des veufs Pierre de Rivon de Budemont et Jean-Baptiste Godefroy. Dans les deux cas, on remarque l'absence de livres au décès de l'épouse. En 1741, dix années après le décès de Marie Godé, Rivon meurt à son tour et laisse cinq ouvrages de toute nature: La Vie des Saints, Le jardinier fleuriste, Les fonctions de tous les offices, Le Code militaire et les Commentaires sur la Coutume de Paris de Claude Ferrière. Le cas de Godefroy est similaire. Le second inventaire est fait deux ans après le premier et nous permet de découvrir 16 livres, dont «neuf vieux Bouquins en anglais non Estimés».

Les pratiques notariales peuvent aussi être mises en cause. Les historiens français ont amplement souligné l'absence des livres de colportage, ceux de la Bibliothèque bleue, dans les actes notariés. Selon eux, les

33. Archives nationales du Québec, Centre de Québec (ANQ-Q), greffe de J.-C. Louet, Inventaire des biens de la communauté de Eustache Chartier, veuf de Marie-Françoise RenautDavaine de Desmeloise, 25 juin 1723. La seule autre mention du genre dans un inventaire concerne le père de Chartier, René-Louis. Le contrat de mariage de Claude de Bermen de Lamartinière disposait aussi d'une clause, où le mari se réservait «ses armes et tous ses livres" advenant le décès de l'épouse.

34. ANQ-Q, greffe de N. Pinguet de Vaucour, Inventaire des biens de feu Eustache Chartier de Lotbinière, 8 mai 1749 . 
notaires de France, "ne mentionnent pas plus que leurs homologues étrangers le nombre de livres de colportage et de dévotion que les imprimeries de province multipliaient alors et que libraires et merciers diffusaient jusque dans les bourgs ${ }^{35}$ ». Pour Daniel Roche, «livres et brochures de culture pratique ou ouvrages de piété sans grande valeur marchande ont pu être soustraits à la prisée du fait même de leur abondance ${ }^{36}$ ". Ces remarques ont même été reprises par le spécialiste de l'histoire du livre aux États-Unis, David D. Hall, qui affirme qu' «en règle générale aucun inventaire ne tient compte des livres "bon marché" - par exemple, les almanachs ne sont jamais mentionné ${ }^{37} »$. Contrairement à la situation européenne ou même américaine, les notaires canadiens semblent leur accorder quelque importance puisqu'ils décrivent parfois des livres en lots. Quarante de ces lots ont été repérés et le nombre de volumes de 35 d'entre eux peut être connu. Mais on peut penser que certains notaires les évacuent des inventaires.

\section{NOMBRE ET IMPORTANCE DES COLLECTIONS}

L'absence de livres demeure donc une donnée majoritaire dans les inventaires après décès. Est-elle compensée par un plus grand nombre d'ouvrages dans les actes qui en font état? Pour cette analyse, seul le nombre de volumes est retenu car le comptage par titres ne donnerait que des résultats fort incomplets. En effet, on recense 442 volumes sans titre dans les inventaires. Par ailleurs, bien que le tome corresponde à la division du contenu d'un ouvrage et que le volume renvoie à la division physique, nous avons fait fi de cette différence puisque, dans les inventaires, les notaires utilisent indistinctement tome et volume dans leurs descriptions. Enfin, les livres faisant partie des fonds de marchandises ont été mis de côté. On en retrouve dans l'inventaire de Charles Aubert (1695), dans celui de Jacques Leber (1706) ou dans celui de Théodose Denis De Vitré. Toutefois, les livres de ce dernier, rangés dans son magasin, ont été retenus. Ces livres, disponibles en un seul exemplaire et dont l'état laisse quelquefois à désirer, semblent indiquer que le magasin était le lieu de conservation des livres personnels de Denis de Vitré. Au total,

35. Henri-Jean Martin et Bruno Delmas, Histoire et pouvoirs de l'écrit, op. cit., 332.

36. Daniel Roche, Le peuple de Paris. Essai sur la culture populaire au XVIII ${ }^{e}$ siècle (Paris, Fayard, 1998), 288.

37. David D. Hall, «Lecteurs et la lecture», dans Roger Chartier, Histoire de la lecture : un bilan des recherches. Actes du colloque des 29 et 30 janvier 1993 (Paris) (Paris, IMEC, 1995), 177, note 7. 
parmi les 96 inventaires avec livres, $93^{38}$ cumulent 743 titres d'ouvrages et 40 lots de volumes sans mention de titres pour un total de 1572 volumes. La moyenne se situe ainsi à près de 17 volumes par inventaire. Pour la sous-période 1670-1700, le nombre moyen de volumes s'élève à 26 et s'explique par la présence de la bibliothèque de Charles Aubert, qui en contient 130 lors de l'inventaire de 1695. Sans celle-ci, le nombre moyen de volumes chute à 14 . Au xviII ${ }^{\mathrm{e}}$ siècle, le nombre moyen est de 16. En affinant un peu plus notre analyse, on remarque qu'entre 1751 et 1764, le faible taux d'inventaires avec livres est compensé par un nombre plus grand de volumes par acte, soit 20. L'augmentation du nombre moyen de volumes durant le XviII $^{\mathrm{e}}$ siècle est aussi confirmée par les études françaises.

Dans l'ensemble des actes répertoriant des quantités de volumes, 31 (33\%) se retrouvent au-dessus de la moyenne globale de 17 volumes. La seule bibliothèque dépassant 100 volumes est celle de Charles Aubert en 1695. Gilles Proulx en recense 17 pour l'ensemble de la population de Québec dont 3 comptant plus de 1000 volumes. Le nombre d'actes comprenant moins de 10 livres s'élève à $47 \%$, comparativement à $60 \%$ pour la ville de Québec. Il faut aussi souligner la présence d'un seul volume dans 13 inventaires. Par conséquent, la place du livre est donc relativement modeste parmi les biens de la noblesse canadienne, encore que supérieure à celle occupée dans les avoirs des citadins de Québec. Avec une moyenne de 17 volumes, la bibliothèque nobiliaire canadienne se situe loin derrière son équivalent français du XvIII ${ }^{\mathrm{e}}$ siècle, qui contient entre 100 et 300 volumes. Elle s'apparente plutôt à celle des écuyers et chevaliers des villes de l'Ouest de la France de la fin du $\mathrm{xvII}^{\mathrm{e}}$ siècle qui compte moins de vingt volumes.

\section{LES LIEUX DE CONSERVATION DU LIVRE}

Le dernier «test» proposé par Daniel Roche porte sur la localisation des livres dans l'habitation. Où se trouvent-ils et comment sont-ils rangés? L'ensemble des auteurs, principalement en France, s'entendent pour dire que le livre n'est pas rangé à un endroit précis de la maison: "dans le concret du quotidien vécu, tel que le laisse apercevoir la lecture en série

38. Parmi les trois actes non retenus ici, ceux de René-Louis Chartier et d'Eustache Chartier ne mentionnent aucun livre car leur bibliothèque leur était réservée en vertu du contrat de mariage. Quant au troisième, celui de Joseph Boucher-Denoix, seule la mention «Livres de médecine» apparaît dans l'acte notarié. 
des inventaires après décès, le livre est partout dans l'intérieur de la maison $^{39}$ ». Le noble canadien, quant à lui, conserve majoritairement ses livres dans une chambre ou un cabinet (86\%) suivi de loin par le grenier et autres lieux tels que la salle, le magasin, la chapelle ou une redoute ${ }^{40}$. Une communauté, celle de Jacques Lepicard Dumesny et Marie-Renée Chorel de Saint-Romain, range même l'Histoire romaine et 30 petits livres dans la cuisine. Si la grande majorité des nobles placent leurs livres en un seul lieu, certains les dispersent en plusieurs endroits. Théodose Denis de Vitré conserve ses livres personnels dans le magasin alors que son épouse en garde quelques-uns dans un cabinet donnant dans sa chambre. Les premiers sont des livres juridiques, des livres de dévotion, un dictionnaire ou même un manuscrit sur la médecine, alors que les seconds sont uniquement des ouvrages de dévotion. Contrairement à la situation française, on rencontre aussi bien des livres de dévotion dans les cabinets que des livres d'usage professionnel dans les chambres.

À l'intérieur de ces différentes pièces, les livres sont rangés, là où la mention existe, à raison de $55 \%$ dans un meuble tel qu'une armoire, un bahut ou un buffet, et de $45 \%$ dans un coffre, une malle, une valise ou même une cassette. Les livres du marquis Charles Dalogny de La Groye sont placés dans le cabinet, à l'intérieur d'une "Armoire En placage contre La muraille ouvrant a deux Battants fermant a Clef», en compagnie de pots, de tasses et même de la coutellerie. On en retrouve aussi dans un tiroir en dehors d'un "petit cabinet en placage de Bois de noyer avec Les pieds tournées ouvrans a deux panneaux fermant a Clef, et vingt deux tiroirs tant dedans que dehors ${ }^{41}$ ». La situation est similaire à celle observée à Besançon au XviII ${ }^{\mathrm{e}}$ siècle :

Le livre est rangé un peu partout, sans ordre, aussi bien dans une armoire au milieu du linge (chez Bruand et Defusy), dans un coffre (chez l'inspecteur des Ponts et Chaussées Trionbrousse) ou dans un meuble spécial. Ce dernier, appelé indifféremment par les huissiers «buffet», "commode», "secrétaire» comportait deux ou quatre portes ajourées soit par des vitres, soit par un grillage en fil de fer ou de laiton ${ }^{42}$.

39. Roger Chartier et Daniel Roche, "L'histoire quantitative du livre», Revue française d'histoire du livre (1977): 494.

40. Les lieux de conservation des deux tiers des inventaires avec livres peuvent être identifiés.

41. ANQ-Q, greffe Jacques Barbel, Inventaire des biens de la communauté de Geneviève Macart et Charles Dalogny de Lagroys, $1^{\text {er }}$ mars 1724.

42. Paul-Marie Grinevald, "Les bibliothèques à Besançon au xvIII ${ }^{\mathrm{e}}$ siècle», dans Louis Trénard, dir., Les bibliothèques au XVIII eiècle (Bordeaux, Société des bibliophiles, 1989), 225. 
Bien que l'on ne rencontre aucune mention d'une bibliothèque dans les inventaires, on ne peut conclure à l'absence totale d'un meuble dédié aux livres puisque l'aide-major Michel-Jean-Hugues Péan «a disposé autour de son cabinet des armoires aux panneaux grillagés de laiton et doublés de taffetas $(1760)^{43}$ " où sont rangés ses volumes. En octobre 1756, madame de Repentigny loue une maison à Devienne qui comprend, dans une chambre à côté de la salle, "une armoire Servant de Bibliotheque ayant vingt quatre Carreau de verre entier fermant avec deux tarjettes, au dessus une armoire ouvrant a deux Panneaux fermant a clef ${ }^{44} »$. Les livres des nobles canadiens se retrouvent donc généralement dans des lieux fermés tels que la chambre et le cabinet. Ils semblent peu visibles et n'ont pas la fonction d'impressionner le visiteur.

Identifier la classification des ouvrages privilégiée par les nobles s'avère une tâche plus complexe. Le classement thématique semble inconnu. Chez Augustin Juchereau, Le guide des négociants est suivi d'un livre de théologie et d'un livre sur le théâtre italien. Néanmoins, nous croyons que les nobles disposant d'un nombre important de volumes les classent en fonction des formats, comme cela a été constaté à Besançon $^{45}$. En reprenant les inventaires des nobles qui comportent un nombre varié de formats, on remarque effectivement que Charles Aubert et Matthieu Collet de Lafortière classent leurs livres de l'in-folio vers l'in-octavo, selon la description faite par le notaire. Les livres in-folio de Louis Rouer de Villeray précèdent ses in-quarto, alors que ceux de Paul Denis de Saint-Simon sont rangés de l'in-quarto au in-douze. Ce type de classement est le plus répandu à l'époque. Seule exception, les in-octavo, in-seize, in-quarto et in-folio de Claude Bermen de Lamartinière sont disposés sans ordre.

\section{LES CATÉGORIES LITTÉRAIRES}

La répartition des livres en catégories littéraires est un élément central de l'histoire de la consommation du livre. Basée sur le plan de classification élaboré par François Furet (1 - théologie, 2 - droit et jurisprudence, 3 - histoire, 4 - sciences et arts et 5 - belles lettres), cette distribution permet de cerner la composition, voire le type de livres privi-

43. "La maison d'Arnoux où Montcalm est mort», Bulletin des recherches historiques, IX,1 (janvier 1903): 4.

44. ANQ-Q, greffe Jean-Claude Panet, 4 octobre 1756.

45. P.-M. Grinevald, "Les bibliothèques à Besançon au XviII ${ }^{\mathrm{e}}$ siècle », op. cit., 226. 
légiés par la noblesse canadienne à partir cette fois-ci des titres des ouvrages contenus dans les inventaires après décès. Les données obtenues témoignent d'une nette domination du livre de théologie, avec une proportion de $42 \%$ des titres recensés ${ }^{46}$. Les quatre autres catégories qui regroupent tout de même plus de la moitié des titres se répartissent de la façon suivante : $17 \%$ pour droit et jurisprudence, $16 \%$ pour science et arts, $15 \%$ pour histoire et seulement $11 \%$ pour belles lettres. Comme le souligne François Melançon, «le monde premier de la lecture dans l'intimité domestique reste celui de la religion ${ }^{47}$ ».

En comparant avec la population d'autres groupes, d'intéressantes différences surgissent (voir le tableau 2). Avec les gens de la ville de Québec, on remarque que la grande différence se situe au niveau des catégories théologie et belles lettres. La population québécoise accorde une plus grande importance aux belles lettres et un intérêt moindre à la théologie. La présence des trois plus importantes bibliothèques de la Nouvelle-France, soit celles de l'intendant Claude-Thomas Dupuy (575 titres répartis en 1045 volumes), du procureur Guillaume Verrier (plus de 2000 volumes) et du marchand François-Étienne Cugnet (797 titres

TABLEAU 2

Répartition des livres en catégories littéraires

\begin{tabular}{lcccc}
\hline & $\begin{array}{c}\text { Noblesse } \\
\text { canadienne } \\
(\mathbf{1 6 7 0 - 1 7 6 4 )} \\
\text { en \% }\end{array}$ & $\begin{array}{c}\text { Population } \\
\text { de Québec } \\
(\mathbf{1 6 9 0 - 1 7 6 0 )} \\
\text { en \% }\end{array}$ & $\begin{array}{c}\text { Noblesse } \\
\text { bretonne } \\
(\mathbf{X V I I I} \text { siècle) } \\
\text { en \% }\end{array}$ & $\begin{array}{c}\text { Noblesse } \\
\text { parisienne } \\
(\mathbf{1 7 5 0 - 1 7 5 9 )} \\
\text { en \% }\end{array}$ \\
\hline Théologie & 42 & 27 & 23 & 22 \\
Droit & 17 & 22 & 17 & 13 \\
Histoire & 15 & 15 & 21 & 35 \\
Science et Arts & 16 & 16 & 14 & 9 \\
Belles-Lettres & 11 & 21 & 24 & 21 \\
\hline
\end{tabular}

Sources pour la population de Québec et les noblesses françaises: Gilles Proulx, Loisirs québécois: des livres et des cabarets 1690-1760 (Québec, Service canadien des parcs, 1987); Histoire des bibliothèques françaises, 1530-1780. Claude Jolly, dir., Histoire des bibliothèques françaises, volume 2: Bibliothèques sous l'Ancien Régime, 1530-1789 (s.l., Promodis, Éditions du Cercle de la Librairie, 1988).

46. Les pourcentages ont été calculés à partir de 711 des 743 titres disponibles dans les inventaires; les 32 restants n'ont pu être classés dans une catégorie.

47. F. Melançon, «Façonner et surveiller l'intime: lire en Nouvelle-France», op. cit., 34. 
répartis en 1539 volumes), permet probablement d'expliquer cette forte présence des belles lettres au détriment du livre religieux. La bibliothèque de Dupuy ne contient que $8 \%$ d'ouvrages religieux contre $26 \%$ d'ouvrages littéraires, alors que celle de Cugnet en comprend $12 \%$ pour la première catégorie et $25 \%$ pour la seconde.

En France, il est généralement admis que l'on assiste au xviII siècle à un changement des comportements nobiliaires: un transfert s'effectue du livre de droit et de religion vers le livre d'histoire et de belles lettres. Chez les nobles canadiens, on observe bien un déclin du livre de droit de même qu'une montée de la littérature et des sciences et arts. Cependant, à l'encontre de l'expérience française, on remarque une stabilité importante du livre religieux et un déclin du livre d'histoire. Le taux élevé de la catégorie théologie s'explique aussi par la présence des prêtres écuyers. René Portneuf (1759) laisse à sa mort 42 livres de dévotion sur 56 titres, tandis que Charles Dufrost de la Jemerais (1750) et François Marganne de Lavaltrie (1750) possèdent respectivement 14 et 6 livres exclusivement reliés à leur ministère. Sans ces derniers, le taux global pour la théologie s'établirait à $37 \%{ }^{48}$.

La première catégorie, théologie, est donc très populaire auprès des nobles canadiens, qui possèdent avant tout des livres de dévotion. Sur les dix-huit titres les plus souvent rencontrés dans les inventaires après décès (voir annexe A), le livre religieux en accapare dix, dont les deux premières positions avec les livres d'heures et la Vie des Saints, qui se partagent le premier rang, et l'Office de la Semaine Sainte au second rang. Le livre d'heures a longtemps été le livre de prière par excellence. Son origine remonte au Moyen-Âge et il était souvent le seul livre conservé et consulté même par les personnes ne sachant pas lire. Ce livre

[...] permettait au fidèle de s'adresser seul, dans le recueillement, à son Créateur. Constitué d'une mosaïque de textes usuels destinés à faciliter l'élévation de l'âme, il comportait aussi quelques indications pratiques éparses

48. Il existe aussi d'importantes bibliothèques nobiliaires qui n'ont pas été intégrées au corpus documentaire et dont la composition est connue, entre autres celle de Thomas-Jacques Taschereau, secrétaire de l'intendant Claude-Thomas Dupuy. En février 1750, lors de son inventaire après décès, on dénombre 184 titres répartis en 339 volumes. La grande majorité de ses titres sont en littérature $(30 \%)$ et en histoire $(29 \%)$. Viennent ensuite les catégories arts et sciences $(16 \%)$, théologie $(13 \%)$ et droit $(12 \%)$. Cette répartition est évidemment à l'opposé de ce qu'on retrouve chez les autres nobles canadiens sauf en ce qui concerne les ouvrages de la catégorie sciences et arts. La place des ouvrages religieux y est moins importante, tandis que les belles lettres et l'histoire dominent largement les autres catégories. Singulière, la bibliothèque de Taschereau annonce le $\mathrm{xIx}^{\mathrm{e}}$ siècle. 
dans le calendrier et dans l'alphabet, notamment des conseils de médecine populaire. Richement illustré, il prolongeait enfin, au fil de ses pages les leçons des sculptures et des fresques de l'église et pouvait être utile grâce à ces images aux membres de la maisonnée qui ne savaient pas lire ${ }^{49}$.

Alberto Manguel souligne aussi que les «livres d'heures devinrent des cadeaux de mariage traditionnels dans la noblesse, et par la suite dans la bourgeoisie riche ${ }^{50} "$, dès le $\mathrm{xv}^{\mathrm{e}}$ siècle. On retrouve ainsi chez les nobles canadiens des Heures de vie, Heures de la Congrégation, Heures à l'usage du diocèse de Paris, Heures à la Reine, Heures royales, etc.

Pour la catégorie suivante, droit et jurisprudence, la majorité des titres concernent le droit civil, la jurisprudence et la pratique, mais pas le droit canon ou ecclésiastique. Sept individus, tous conseillers du roi, possèdent $85 \%$ des titres de cette catégorie (96 sur 113). Les différentes ordonnances royales et la Coutume de Paris détiennent respectivement les troisième et cinquième places du palmarès des titres les plus populaires (voir annexe A). Parmi les six grandes ordonnances du règne de Louis XIV ${ }^{51}$, seules deux ne figurent pas dans les inventaires après décès: celle des eaux et forêts (1669) - qui ne concerne pas le Canada - et celle de la police des îles d'Amérique ou Code noir $(1685)^{52}$. Parmi les quatre autres, les plus fréquemment rencontrées sont celles de la marine (1681) et celle de la justice (1667).

En ce qui concerne la troisième catégorie, l'histoire, on constate sa baisse continuelle tout au long de la période, ce qui va à l'encontre de la tendance nobiliaire française. L'histoire moderne de la France de même que celles d'autres pays comme la Hollande ou d'autres civilisations telles que la Turquie ou même l'Histoire romaine de Charles Rollin sont plus présentes que l'histoire du Canada. Déjà en 1930, Antoine Roy avançait l'hypothèse que «ce qui les [les Canadiens sous le Régime français] attirait bien plus que l'histoire de leur propre pays, c'était celle des pays lointains ${ }^{53}$ ». On ne relève que deux titres en histoire du Canada. Le

49. Henri-Jean Martin et Bruno Delmas, Histoire et pouvoirs de l'écrit (Paris, Albin Michel, 1996), 337.

50. A. Manguel, Une histoire de la lecture, op. cit., 160.

51. Elles sont indiquées dans les inventaires par leur année ou par leur sujet.

52. Le Code noir de 1685, qui a pour véritable titre Ordonnance du Roi Concernant la discipline de l'Eglise, et l'état et qualité des negres esclaves, aux îles de l'Amérique, régit l'esclavage dans les colonies françaises des Antilles. En Nouvelle-France, c'est le 13 avril 1709 que l'esclavage est réglementé par l'enregistrement de l'Ordonnance rendue au fuget des Negres et des Sauvages appelés Panis.

53. Antoine Roy, Les lettres, les arts et les sciences au Canada sous le régime français (Paris, Jouve, 1930), 77. 
premier, l'Histoire de l'Amérique, est l'ouvrage de Claude-Charles Le Roy Bacqueville de La Potherie paru en 1722 sous le titre de Histoire de l'Amérique Septentrionale et que détient Jacques-Hugues Péan en 1747. Le second, identifié comme une histoire du Canada en latin, appartient à René Portneuf $(1760)^{54}$. Aucune trace de l'Histoire et description générale de la Nouvelle-France du père Pierre-François-Xavier de Charlevoix ou des Relations des jésuites qui, selon Roy, "ne paraissent pas avoir joui alors d'une grande vogue ${ }^{55} »$. Les seuls autres titres concernant le Canada ou l'Amérique sont: La vie de la vénérable Mère Marie de l'Incarnation... de Claude Martin, un Rituel du Diocèse de Québec de $\mathrm{M}^{\mathrm{gr}}$ de Saint-Vallier, Description de la Louisiane... du père Louis Hennepin et les Voyages du baron de Lahontan dans l'Amérique septentrionale de Louis Armand de Lom d'Arce, baron de Lahontan.

La catégorie "sciences et arts" est en forte croissance dans la noblesse canadienne. De $6 \%$ au $\mathrm{XvII}^{\mathrm{e}}$ siècle, elle passe à $25 \%$ pour les deux dernières décennies de notre période. Les sous-catégories en vogue sont les sciences, la philosophie, les arts libéraux et l'économie politique. Par contre, l'agriculture, les arts mécaniques et les arts spécialisés sont peu représentés. En sciences, les nobles favorisent avant tout les mathématiques, qui englobent aussi l'astronomie et la science de la navigation, suivi de la médecine. Henri-Jean Martin ne souligne-t-il pas qu' «au temps de la révolution mécaniste, les officiers de l’armée royale française commençaient à s'initier aux mathématiques et en particulier à la géométrie $\left.{ }^{56}\right)$ ?

La physique ne semble pas les attirer. Jean-Claude Dubé souligne aussi que les "Canadiens semblaient peu intéressés par les traités de physique» de l'intendant Dupuy puisque aucun ne trouve preneur lors d'une vente en octobre 1730. Dupuy demande alors que ces livres soient transportés à Rochefort pour y être vendus ${ }^{57}$. Les titres en sciences naturelles sont aussi peu nombreux. Pierre André de Leigne (1735) possède un Cours de chimie, tandis que Joseph Dubois Berthelet de Beaucours (1759) possède une Dissertation sur les reptiles et une Histoire des faussiles.

54. Il s'agit probablement de Historiae canadensis, seu Novae Franciae libri decem du père François Ducreux et paru en 1664, la même année que Histoire véritable et naturelle... de Pierre Boucher.

55. A. Roy, Les lettres, les arts et les sciences au Canada sous le régime français, op. cit., 77.

56. Martin et Delmas, Histoire et pouvoirs de l'écrit, op. cit., 339.

57. Dupuy en a-t-il laissé à son secrétaire, Thomas-Jacques Taschereau? Ce dernier conserve deux ouvrages de physique à sa mort, dont un manuscrit. 
Depuis 1685, les nobles peuvent commercer sans déroger en NouvelleFrance. Les ouvrages d'économie politique sont donc très appréciés et plus particulièrement Le parfait négociant ou Instruction générale pour ce qui regarde le commerce des marchandises de France \& des pays étrangers de Jacques Savary qui occupe le septième rang des titres les plus rencontrés en compagnie de la Bible, du Bréviaire romain et des catéchismes (voir annexe A). En ce qui concerne le mouvement des Lumières, on remarque que les nobles ne possèdent aucun des grands livres publiés à partir de 1748, année où paraît De l'Esprit des lois de Montesquieu. Aucune trace de Histoire naturelle générale et particulière du comte de Buffon (à partir de 1749), du Siècle de Louis XIV de Voltaire (1751), du premier tome de L'Encyclopédie (1751) ou du Discours sur l'origine et les fondements de l'inégalité parmi les hommes de Jean-Jacques Rousseau (1755).

La place des belles lettres, la cinquième et dernière catégorie, est très modeste pour l'ensemble de la période. Cette catégorie n'obtient que $6 \%$ au XVII siècle pour culminer à $13 \%$ entre 1751 et 1765 . La préférence des nobles canadiens va majoritairement à la poésie (57\%), suivie de très loin par les dictionnaires $(18 \%)$ et les ouvrages de grammaire (12\%). Les nobles optent pour la poésie avant même le roman ou l'art dramatique. Ovide et Virgile sont les poètes dont les œuvres sont les plus mentionnées. Si la poésie s'étale sur l'ensemble de la période, on remarque que l'art dramatique n'apparaît dans les inventaires qu'à partir de 1715 , alors que le roman n'est présent que vers 1720 . Tout comme à Québec entre 1690 et 1760, Don Quichotte de la Manche de Cervantès est le roman le plus populaire et occupe le huitième rang du palmarès des titres (voir annexe A). Parmi les treize dictionnaires, on remarque sept dictionnaires de langues, principalement de langue latine, mais aussi un dictionnaire latin-grec chez Augustin Juchereau, un dictionnaire françaisbreton chez Joseph Dubois Berthelet de Beaucours, de même qu'une grammaire et un dictionnaire français-espagnol chez Mathieu Collet de Lafortière. Cependant, les nobles ne possèdent aucun dictionnaire de langues orientales comme on en retrouvait chez l'intendant ClaudeThomas Dupuy qui, avec ses traités de physique, les rapatria à Rochefort.

Pour terminer, il n'est pas inintéressant d'examiner la langue des ouvrages. On remarque en effet la présence de livres latins, qui comptent pour $7 \%$ de l'ensemble des titres ${ }^{58}$, alors que l'apport des autres

58. Plus de la moitié de ce taux renvoie à des ouvrages bilingues (français-latin). 
langues est marginal. Mis à part les dictionnaires grec, breton ou espagnol, on ne rencontre qu'un recueil de poésies en latin-italien-castillan chez Curault-Gourville de Saint-Michel (1748) et une grammaire italienne chez le marquis d'Alogny de la Groye (1724). On ne trouve aucun livre en langue anglaise avant $1762^{59}$. Nous savons néanmoins que Mme Bégon a eu entre les mains des "gazettes anglaises" qu'elle traduisait pour le gouverneur de La Galissonnière ${ }^{60}$.

\section{LES «SIGNES DE LECTURES " DANS LA NOBLESSE CANADIENNE ET USAGES DES LIVRES}

La recherche d'indices ou de «signes de lecture» permet de combler les lacunes de l'analyse quantitative du livre relativement aux pratiques individuelles ou collectives de lecture. Toutefois, cette quête est hasardeuse. Les signes de lecture peuvent figurer dans la correspondance, dans les documents judiciaires, dans les testaments ou même directement dans les livres. L'achat de livres lors des ventes de biens peut aussi donner des indices. Mais les nobles achètent peu de livres ${ }^{61}$. Quant au prêt de livres, on en trouve peu de traces dans les inventaires ${ }^{62}$. Nous en sommes réduits à quelques exemples d'indices de lecture. Tout en étant conscient des limites de cet exercice, demandons-nous si les membres de la noblesse canadienne font un usage utilitaire (besoins religieux et besoins professionnels) ou ludique de leurs livres.

L'usage de livres pour les besoins religieux ne semble faire aucun doute vu l'importante proportion de ceux-ci dans la noblesse canadienne. Le sulpicien Pierre Rémy souhaite, à sa mort, léguer une Vie des Saints à Pierre de Saint-Ours et à sa femme "pour leur estre donné apres

59. Ce sont les «neuf vieux Bouquins en anglais» de Jean-Baptiste Godefroy. ANQ-M, greffe de Pierre Panet de Méru, Inventaire des biens de feu Jean-Baptiste Godefroy et de Marquerite de Couagne, 6 septembre 1762 .

60. «La correspondance de Madame Bégon 1748-1752» In Rapport de l'archiviste de la province de Québec pour 1934-1935 (Québec, Département du secrétaire de la province, 1935), 213 (23 avril 1749).

61. Le rachat, tout aussi exceptionnel, peut toutefois être un indice de l'importance que des individus accordent à un ouvrage. Louis-Odet Piercot de Bailleul achète l'Histoire turque lors de la vente des biens de sa propre communauté. Louis Liénard de Beaujeu de Villemomble semble faire de même lorsqu'il achète une Semaine Sainte.

62. Parmi ses livres, le curé René Portneuf conservait neuf titres appartenant au Séminaire de Québec, probablement des livres empruntés. Dans un autre inventaire après décès, Gertrude Legardeur, veuve de Jean-Baptiste Céloron de Blainville admet avoir en sa possession le «...Memorial de la vie Chrestienne Sur lequel Est Ecrit Le nom de Mr Ganneau que la Dame a declare avoir Empreste», ANQ-Q, Collection de pièces judiciaires et notariales..., nº 1699, date inconnue. 
ma mort a dessin qu'ils puissent en faire tous les Jours leur lecture spirituelle ${ }^{63}$ ». Dans son testament, Pierre Boucher recommande à «ceux qui sont dans lestat du mariage lissé la famille sainte du R. P. Cordier jesuiste les conseilles de la sagesse et autres semblables ${ }^{64}$ ».

L'usage de livres pour des besoins professionnels est aussi indéniable. Mathieu Collet, qui dispose de 30 volumes principalement de nature juridique, donnait des cours de droit dès le début du xviII ${ }^{e}$ siècle, alors qu'il était procureur général. Le livre sert aussi à la défense ou à l'avancement de causes. Dans le procès qui l'oppose à la veuve Hertel de Rouville au sujet du mariage de leurs enfants, Pierre André de Leigne cite un ouvrage juridique pour sa défense:

Il est vray que M. Argoud, célèbre auteur, dit que les mineurs de 25 ans, ne peuvent contracter mariage, sans le consentement de leurs père et mère, tuteur et curateur, et que cependant de défaut de consentement n'emporterait pas nullité de mariage ${ }^{65}$.

Gaspard Chaussegros de Léry père fera de même auprès du Conseil de Marine en 1723 pour faire valoir ses droits:

Il y a huit ans que j'ai l'honneur de servir dans cette colonie, l'ordonnance du dernier fevrier, Tom IV, page 45, porte qu'on portera l'ordre aux ingénieurs et par la commission de Capitaine dans les troupes de cette colonie que Sa Majesté m’a accordée en 1720, on doit le faire, comme cela ne m’est pas arrivé et que je m'aperçois que c'est par mépris, je vous supplie très humblement ${ }^{66} \ldots$

L'usage de la lecture dans les moments de loisirs est probablement exclusif aux élites, mais les exemples sont rarissimes. On y voit Mme Bégon se faire lire une tragédie de Corneille par sa petite-fille ou Nicholas-Roch de Ramezay écrire à sa femme Marie-Louise Godefroy de Tonnancour le 14 avril 1764 de Paris: "Mais que tout cela nous fera-t-il rien quand nous serons chez nous ensemble, une fois rangés, nous nous

63. Archives nationales du Québec, Centre de Montréal (ANQ-M), greffe Michel Laferté Lepailleur, 20 mai 1720.

64. "Les adieux ou dernières volontés de Pierre Boucher», Rapport de l'archiviste de la province de Québec pour 1921-1922, 1922, fac-similé, 14 p.

65. Cité dans «La famille André de Leigne», Bulletin des recherches historiques, XLI,7 (juillet 1935): 396.

66. Lettre de M. Chaussegros de Léry au Conseil de Marine, 30 septembre 1723 in Inventaire des papiers de Léry, I: 95. 
moquerons de toute la terre et lirons la Gazette où nous jugerons, nous condamnerons, comme bon nous semblera, les prévarications ${ }^{67} \ldots$ »

On peut aussi être tenté de faire un lien entre l'usage des livres et la présence d'objets spécifiques. La possession d'une paire de lunettes a-telle un lien direct avec la lecture? Il arrive parfois que le lien soit plus évident comme chez François Curault-Gourville de Saint-Michel qui possède des livres de musique et qui dispose aussi d' "un orgue portatif avec Sa [...] Souffelets et tuyau en mauvais etat» valant 50 livres $^{68}$. Les traités de médecine de l'écuyer et chirurgien Joseph Boucher-Denoix sont compris dans une liste de produits médicaux. Louis Laporte de Louvigny, gouverneur de la ville des Trois-Rivières, possède un «compas de proportion de cuivre jaune garnie de Son pad et ses quatre pinules (8 livres)» et un " quadran solaire garnyes de Son Etuy (10 livres) $)^{69}$ " qui accompagnent, entre autres, ses "onze tôme de livres reliés en veau de divers auteurs tant sur la géometrie que sur la navigation ${ }^{70} »$. Finalement, les instruments possédés par Augustin Juchereau de Maure (arbalestrille ${ }^{71}$ et compas) sont en lien avec certains des livres qu'il possède tels que ses ouvrages de navigation et de mathématiques.

La présence d'images ou de peintures assure-t-elle la possession de livres? Dans les œuvres exécutées pour les nobles canadiens, une place est-elle accordée au livre comme chez les nobles de France où il «est toujours présent dans le portrait d'apparat que la noblesse commande aux peintres, le plus souvent il magnifie l'emprise sur le monde que donnent, dans la retraite du cabinet de travail, les livres lus ou écrits ${ }^{72}$ »? Sur une dizaine de portraits de nobles canadiens que nous avons identifiés et qui subsistent aujourd'hui dans des musées québécois, aucun ne montre un individu avec un livre ${ }^{73}$. Les portraits présentent généra-

67. Cité par Pierre-Georges Roy, «M. de Ramesay, lieutenant du Roi à Québec, après 1759 », Bulletin des recherches historiques, XXII,12 (décembre 1916): 360-361.

68. ANQ-M, greffe L.-C. Danré de Blanzy, Inventaire des biens de la communauté de François Curault-Gourville de Saint-Michel et de Perrine Lefebvre-Duplessy-Faber, 19 septembre 1748.

69. ANQ-Q, greffe Jacques Barbel, Inventaire des biens de la communauté de Marie Nolan, veuve de Louis de Laporte Louvigny, 7 juin 1727.

70. Ibid.

71. Cet instrument, en forme d'arbalète, est apparu au $x{ }^{e}$ siècle. Il servait à mesurer la latitude.

72. Roche, Les Républicains des lettres: gens de culture et Lumières au XVIII ${ }^{e}$ siècle (Paris, Fayard, 1988), 94.

73. Quelques personnes n'appartenant pas à la noblesse sont toutefois peints un livre à la main : Michel Sarrazin, $M^{\mathrm{gr}}$ Pierre-Herman Dosquet, Mère Louise Soumande de Saint-Augustin et le récollet Emmanuel Crespel. 
lement des bustes et valorisent, pour les hommes, l'habillement et les décorations militaires, plus particulièrement la croix de Saint-Louis. Par contre, un ex-voto peint vers 1696 et conservé au musée de sainte Anne à Sainte-Anne-de-Beaupré nous montre Pierre Le Moyne d'Iberville agenouillé aux pieds de sainte Anne et lui présentant un document et un livre ouvert sur une écritoire à la suite de l'expédition de 1694 à la Baie d'Hudson ${ }^{74}$.

\section{CONCLUSION}

L'analyse quantitative de la présence du livre chez les nobles canadiens permet de constater que $38 \%$ de leurs inventaires contiennent des livres, un pourcentage supérieur à l'ensemble de la population de la ville de Québec, mais inférieur à celui des noblesses françaises; qu'après un accroissement de ce pourcentage entre 1701 et 1750, on assiste à une baisse importante à la fin du Régime français; et, qu'on ne peut faire de lien entre la présence de livres ou l'importance des collections et le degré élevé de fortune, alors qu'il est possible de l'établir avec la profession. Nous avons aussi observé que la chambre et le cabinet sont les lieux de conservation et de rangement favorisés par les nobles. Finalement, la répartition en catégories littéraires indique une forte domination du livre religieux au détriment des belles lettres, un déclin du livre juridique et du livre d'histoire, mais aussi une poussée importante des ouvrages de la catégorie "sciences et arts".

Il est indéniable que l'étude des inventaires pour déceler la présence du livre chez les nobles canadiens est essentielle à la connaissance des pratiques culturelles du groupe, ne serait-ce qu'en raison du nombre limité de sources et d'informations sur ce sujet. La recherche de signes de lectures est fort intéressante, mais elle reste incertaine et ne peut être accomplie que sur une longue période. L'étude des images et peintures que l'on retrouve dans les inventaires après décès pourrait par exemple nous donner de bonnes hypothèses sur ces pratiques, en raison de l'importance de l'imagerie religieuse et du nombre non négligeable de portraits familiaux et royaux. Mais encore faut-il user de beaucoup de circonspection dans leur interprétation.

À l'encontre des opinions exprimées par la plupart des historiens, de Francis Parkman à Christopher Moore, il appert que les nobles se

74. Nous tenons à remercier Patricia Fleming de la Faculty of Information Studies de l’Université de Toronto de nous avoir fait découvrir ce tableau. 
distinguent de l'ensemble de la population de la Nouvelle-France. Ils sont familiers avec l'écrit, ont un taux de présence du livre plus élevé que celui de la population de la ville de Québec, préfèrent les livres religieux et font un bon accueil aux livres de sciences et d'art. Les nobles canadiens savent aussi utiliser les livres pour leurs besoins. Plus de $75 \%$ des nobles qui sont conseillers royaux détiennent personnellement des livres de nature professionnelle, alors qu'il existe une collection de livres au Conseil supérieur; et certains nobles comme André de Leigne ou Chaussegros de Léry s'en servent pour défendre leurs intérêts personnels ou familiaux. Bougainville soutenait qu'une société littéraire, probablement un cabinet de lecture, s'était formée à Québec. Il y a lieu de croire que certains nobles pouvaient s'y retrouver.

Néanmoins, en Nouvelle-France, on ne retrouve pas de grandes bibliothèques nobiliaires comme en France à cette époque. Les nobles canadiens n’appartiennent vraisemblablement pas à la "République des Lettres». Certains font quand même office de rouages importants pour la connaissance de l'histoire naturelle du Canada. Pensons à l'ingénieur Michel Chartier de Lotbinière qui effectue des observations de latitudes avec le père jésuite Joseph-Pierre de Bonnécamps et avec qui il détermine de façon plus précise la longitude de Québec; à Daniel-Hyacinthe Liénard de Beaujeu, commandant du fort Niagara, qui recueille des plantes à la demande du marquis Rolland-Michel Barrin de La Galissonière, gouverneur de la Nouvelle-France de 1747 à 1749; ou à l'ingénieur Gaspard Chaussegros de Léry à qui La Galissonnière fait parvenir son manuel d'instruction, rédigé avec l'académicien Henri-Louis Duhamel du Monceau et intitulé Avis pour le transport par mer des arbres, des plantes vivaces, des semences, des animaux et de différents autres morceaux d'Histoire Naturelle, pour que Chaussegros l'utilise dans son travail ${ }^{75}$.

À ce stade, on ne peut donc en arriver à des conclusions définitives sur la culture nobiliaire. On ne pourra véritablement apprécier le niveau de culture de la noblesse canadienne qu'en la comparant à l'autre groupe élitaire de la société coloniale: la bourgeoisie canadienne. 
ANNEXEA

Titres les plus rencontrés chez les nobles canadiens

\begin{tabular}{|c|c|c|}
\hline Rang & Titre & Nombre \\
\hline \multirow[t]{2}{*}{1} & Heures & 25 \\
\hline & Vie des saints (+ Abrégés de la...) & 25 \\
\hline 2 & Semaine Sainte (+ Office de la...) & 24 \\
\hline 3 & Ordonnances & 23 \\
\hline 4 & Histoire de France & 17 \\
\hline \multirow[t]{2}{*}{5} & Coutume de Paris & 13 \\
\hline & Dictionnaire & 13 \\
\hline \multirow[t]{2}{*}{6} & Nouveau Testament (+ Testament nouveau) & 11 \\
\hline & Imitation de Jésus Christ & 11 \\
\hline \multirow[t]{4}{*}{7} & Bible & 8 \\
\hline & Bréviaire romain & 8 \\
\hline & Catéchisme & 8 \\
\hline & Parfait négociant & 8 \\
\hline \multirow[t]{4}{*}{8} & Don Quichotte & 5 \\
\hline & Histoire romaine & 5 \\
\hline & Psaumes de David & 5 \\
\hline & Guide des pécheurs & 5 \\
\hline 9 & Grammaire & 4 \\
\hline
\end{tabular}

